\title{
A male with trisomy 9 mosaicism and maternal uniparental disomy for chromosome 9 in the euploid cell line
}

\author{
L R Willatt, B C Clare Davison, D Goudie, J Alexander, H M Dyson, P E Jenks, \\ $M$ E Ferguson-Smith
}

\begin{abstract}
We describe a 17 year old male with a low level of trisomy 9 mosaicism. Maternal uniparental chromosome 9 disomy in the euploid cell line was shown to have arisen after postzygotic loss of the paternal chromosome 9 from the trisomic cell line by cytogenetic and molecular analysis. This is believed to be the first report of uniparental disomy for chromosome 9.

In four of the 11 reported cases of mosaic trisomy 9 syndrome, including our patient, a maternally derived pericentric inversion of the heterochromatic area of chromosome 9 has been present in duplicate in the trisomic cell line. This may have implications for the counselling of patients with this common chromosomal variant.

( $\mathcal{F}$ Med Genet 1992;29:742-4)
\end{abstract}

Mosaic trisomy 9 syndrome is a rare condition with only 11 reported cases. ${ }^{1}$ We describe a patient with trisomy 9 mosaicism and a number of unusual features.

\section{Case report}

A male infant was born at 34 weeks' gestation after a normal spontaneous delivery to healthy unrelated parents (paternal age 35, materna: age 30). The pregnancy was normal although the mother noticed decreased fetal movements. Dysmorphic features were noted at birth and chromosome studies carried out at that time were said to be normal. The patient (fig 1) was referred to us for genetic evaluation at 17 years of age. He was mentally retarded with slow and somewhat indistinct speech. Physical examination showed: weight $44.4 \mathrm{~kg}$ ( 3 rd centile), height $163 \mathrm{~cm}$ ( 3 rd centile), and head circumference $55.5 \mathrm{~cm}$ (50th centile). Craniofacial features included facial asymmetry, short neck, micrognathia, and low set ears. Kyphoscoliosis, inability to extend the elbows fully, tight hamstrings, and knock knees were also present. Apart from mild myopia, there was no eye abnormality. On $x$ ray there were 13 pairs of ribs, abnormal $T 6,7,8,9$ thoracic vertebrae, tibiofemoral subluxation, chronic lateral dislocation of the patella, and evidence of osteochondritis.

\section{CYTOGENETIC STUDIES}

Lymphocytes from peripheral blood were cultured by standard techniques. Of the 100 cells

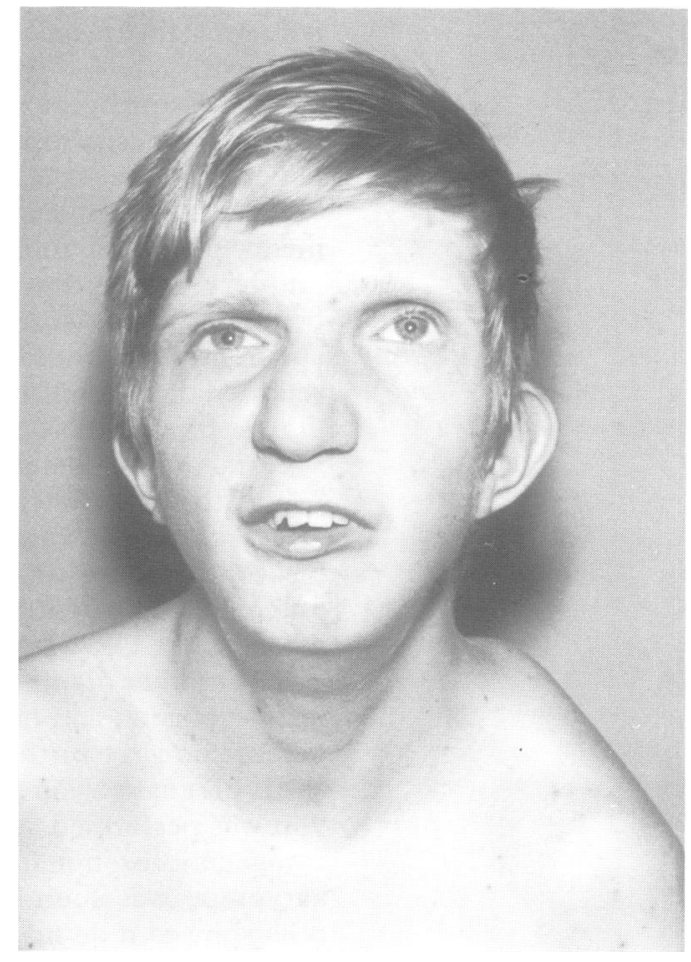

Figure 1 The proband aged 17 years. The facial asymmetry, short neck, and low set ears are evident.

examined, 93 had 46 chromosomes and seven had 47 chromosomes with an extra chromosome 9. GTG and CBG banding showed the presence of a pericentric inversion of the heterochromatic region of chromosome 9 (p11q12) in both chromosomes 9 in the euploid cells and in two of the three chromosomes 9 in the trisomic cells (fig 2).

At elective knee surgery, a further blood sample and muscle and skin biopsies were obtained for chromosome studies. Analysis of the second blood sample confirmed the earlier finding (6/100 cells with trisomy 9). However, chromosome analysis of cultures from the muscle and skin biopsies failed to confirm the mosaicism. One hundred cells were examined from each cultured tissue and these were all $46, X Y$, with homozygosity for the pericentric inversion of chromosome 9 (table 1). Parental chromosome analysis showed that the inverted chromosome 9 was maternal in origin and that the father's chromosomes were normal (fig 2).

A cell line has not been established from this patient. 


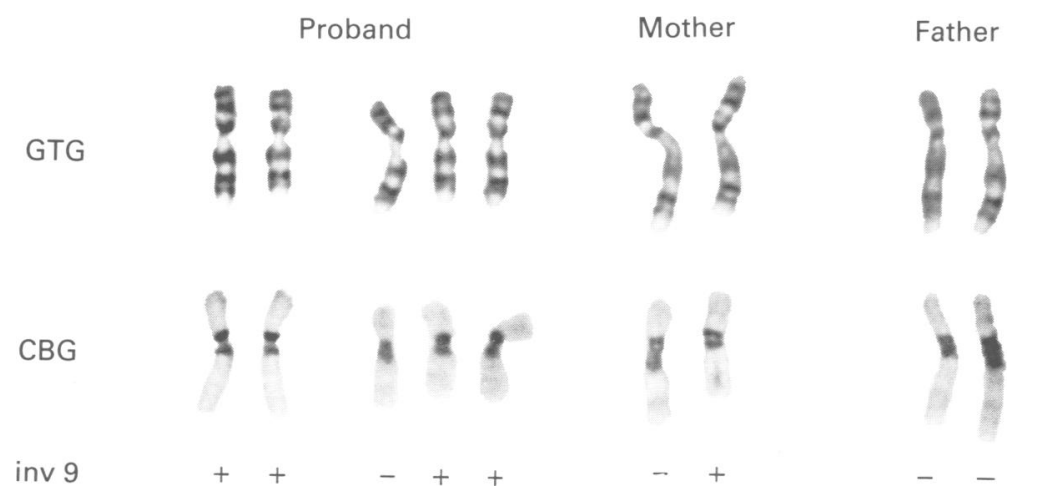

Figure 2 Partial karyotypes showing chromosome 9 homologues (GTG banding above, CBG banding below) from the proband, his mother, and his father.

DNA ANALYSIS

Maternal uniparental disomy for chromosome 9 in the predominant (euploid) cell line was confirmed using polymorphic DNA markers. The maternal and paternal contributions to the patient at the loci listed in table 2 could be distinguished by the greatly reduced intensity or absence of signals from paternally derived DNA fragments (fig 3, top and bottom) consistent with the low level of mosaicism for the trisomic cell line.

Fig 3 shows that the proband has inherited both maternal alleles at D9S53 (9q31) which, in conjunction with the cytogenetic findings, indicates that a recombination must have occurred between D9S53 and the centromere in maternal meiosis $I$ and that duplication of the maternal chromosome 9 could not have resulted from postzygotic non-disjunction. This is reinforced by the findings for $D 9 S 1$, D9S109, D9S127, and 9CMP4 (table 2) which are consistent with a double contribution of maternal alleles and little or no allelic contribution from the father.

Table 1 Cytogenetic findings.

\begin{tabular}{lcc}
\hline & \multicolumn{2}{c}{ No of cells } \\
\cline { 2 - 3 } Cultured tissue & $46, X Y, \operatorname{inv}(9), \operatorname{inv}(9)$ & $47, X Y,+9, \operatorname{inv}(9)$ inv(9) \\
\hline Blood sample 1 & 93 & 7 \\
Blood sample 2 & 94 & 6 \\
Skin & 100 & 0 \\
Muscle & 100 & 0 \\
\hline
\end{tabular}

Table 2 Results of DNA analysis. Observed polymorphic DNA fragments (dinucleotide repeats and RFLPs) derived from parental DNA are numbered sequentially according to size for each locus.

\begin{tabular}{llcccc}
\hline Locus & Probe/enzyme & Location & Father & Mother & Proband \\
\hline D9S6 & EFD126.3/TaqI & $9 \mathrm{q} 34$ & 1,2 & 2 & $1^{*}, 2$ \\
ORM & pa1AGP2/HincII & $9 \mathrm{q} 32$ & 1,2 & 1 & 1 \\
ALAD & pKT218-ALAD/RsaI & $9 \mathrm{q} 32$ & 1,2 & 2 & $1^{*}, 2$ \\
$D 9 S 29$ & Lamp92/TaqI & $9 \mathrm{q} 31$ & 1,2 & 1 & $1,2^{*}$ \\
$D 9 S 53$ & PCR & $9 \mathrm{q} 31$ & 1,3 & 2,4 & $2,4 \dagger$ \\
$D 9 S 109$ & PCR & $9 \mathrm{q} 31$ & 1,2 & 1,3 & $1,3 \dagger$ \\
D9S127 & PCR & $9 \mathrm{q} 31$ & 1,3 & 2,3 & $2,3 \dagger$ \\
D9S1 & HF12-8/TaqI & 9 & 1,2 & 1,2 & $1,2 \dagger$ \\
9CMP4 & PCR & $9 \mathrm{p}$ & 2,3 & 1,3 & $1,2^{*}, 3 \dagger$ \\
9CMP3 & PCR & $9 \mathrm{p}$ & 1,2 & 2 & $1^{*}, 2$ \\
\hline
\end{tabular}

* Polymorphic DNA fragments of reduced intensity on autoradiographs. As a result of the low level of mosaicism for cells containing a paternal chromosome 9 , fragments contributed to the proband by the father alone were of greatly reduced intensity. The remaining polymorphic fragments must have been contributed by the mother (with a small paternal contribution in some cases).

$\dagger$ Loci where there is heterodisomy for the maternal chromosome 9 owing to recombination with the centromere in maternal meiosis.

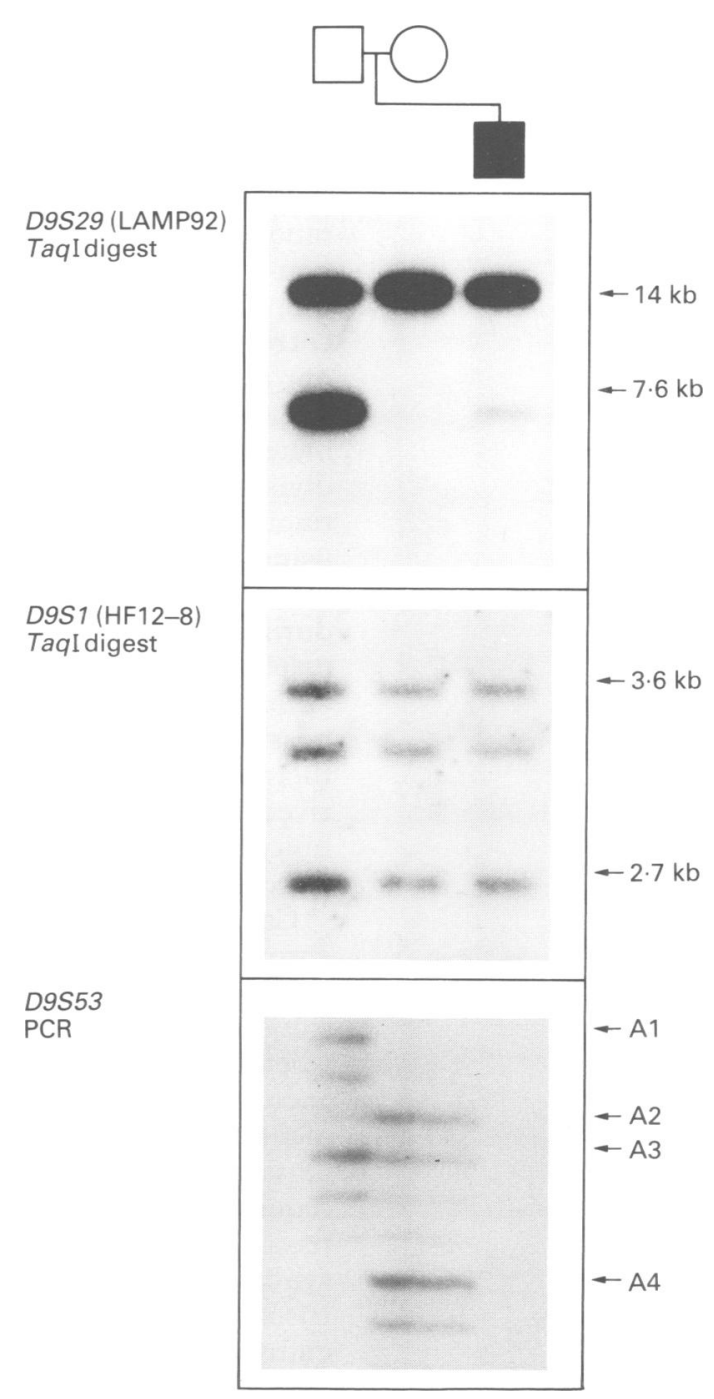

Figure 3 Autogradiographs of polymorphic DNA fragments (indicated by arrows) detected at D9S29( LAMP92), D9S1 (HF12-8), and D9S53 (mfd135) in the proband and his parents. Top and middle: Southern blots of TaqI digested genomic DNA separated on a $0.8 \%$ agarose gel, probed with LAMP92 (D9S29) (top) and HF12-8 (D9S1) (middle). Sizes of polymorphic fragments are indicated. Bottom: PCR amplification of a polymorphic dinucleotide repeat sequence $(D 9 S 53)$ in presence of ${ }^{32} P d C T P$. DNA fragments separated on a $0.6 \%$ polyacrylamide gel. fragments separated on a $0.6 \%$ polyacrylamide gel.
Each allele is represented by two adjacent bands. Polymorphic DNA fragments contributed to the proband by the father alone are undetectable (D9S53 alleles $A 1$ or A3) or greatly reduced in intensity (D9S29 7.6 kb fragment) consistent with the low level of mosaicism for cells containing a paternal chromosome 9. The polymorphic D9S1 fragments in the proband are of equal intensity indicating that they are both predominantly maternal in origin, as are the D9S53 alleles $A 2$ and $A 4$.

\section{Discussion}

To our knowledge, this patient is the first reported case of maternal uniparental disomy for chromosome 9. Furthermore, in this case we were able to determine the mechanism of origin of the uniparental disomy because of the presence of (1) low level mosaicism for a trisomic cell line, (2) a pericentric inversion of both chromosome 9 homologues in the euploid cell line, and (3) evidence provided by DNA analysis of a recombination between the maternal chromosome 9 homologues at meiosis I.

Two mechanisms have been proposed for the origin of uniparental disomy: (1) the loss or 
gain of a chromosome from a trisomic or monosomic conceptus (aneuploid correction) and (2) the fertilisation of a gamete disomic for a chromosome by a gamete nullisomic for the same chromosome (gamete complementation). ${ }^{2}$ Both of these mechanisms require two non-disjunctional events. In cases of maternal uniparental disomy arising from aneuploid correction of a trisomic conceptus (as found in our patient), the first non-disjunctional event may have occurred at maternal meiosis I, or maternal meiosis II, or a very early postzygotic division. Meiosis I errors can only be distinguished from meiosis II errors reliably using polymorphisms or heteromorphisms which do not recombine with the centromere during meiosis. In our case, the finding of a maternally derived pericentric inversion in both chromosome 9 homologues excluded the possibility of a maternal meiosis I error. Furthermore, the presence of two maternally derived alleles at several loci excluded the possibility of postzygotic duplication of the maternal chromosome 9.

Cases of maternal uniparental disomy for chromosomes $4,7,14,15$, and 22 have been reported. ${ }^{3}$ No mosaicism was detected in these subjects so it was not possible to determine whether uniparental disomy had arisen by aneuploid correction or by gamete complementation. As illustrated by our case, low level mosaicism may be difficult to detect and may require the examination of more than one tissue. Two other cases of mosaic trisomy 9 syndrome have been described in which no trisomy 9 cells were found in skin biopsy cultures $^{45}$ and there are numerous published reports of mosaicism confined to a single tissue.

Although the effects of uniparental disomy and imprinting are well defined in mice, there is relatively little information on the effect of uniparental disomy in humans. ${ }^{6}$ The clinical significance of the uniparental disomy in our patient is unclear. His clinical problems, of growth and psychomotor retardation, may have been caused by (1) the unmasking of a recessive condition owing to isodisomy for part of chromosome 9, (2) an imprinting effect, or (3) the presence of the trisomic cell line, or by a combination of these mechanisms. Facial asymmetry may be attributable to mosaicism for the trisomic cell line. Our patient is the oldest reported case of trisomy 9 mosaicism and the low level of mosaicism could account for his relatively mild phenotype.

Of 11 reported cases ${ }^{1}$ of trisomy 9 mosaicism, including our patient, there have been four (three boys and one girl) in which one of the chromosomes 9 had a pericentric inversion of part of the heterochromatic region of chromosome 9.457 In all four of these cases the inverted chromosome 9 was maternal in origin, and the trisomic cell line contained two chromosomes 9 with the inversion. However, in the other three cases, ${ }^{457}$ the euploid line contained only one chromosome 9 with the pericentric inversion, so it was not possible to confirm whether the trisomy 9 cell line had arisen prezygotically or postzygotically (with loss of the monosomy cell line). While these may be coincidental findings and subject to ascertainment bias, the connection between the presence of this inversion and an increased tendency to non-disjunction of chromosome 9 is strongly suggested.

We wish to thank Dr R Miles, Mr A Moore, Mr M Mathewson, and Mr T Vaughan Lane for examining this patient, Dr R Furlong and $\mathrm{Mr} \mathrm{J}$ Lyall for the PCR primers, and Professor $M$ A Ferguson-Smith and Dr E Maher for valuable advice.

1 Diaz-Mares L, Molina B, Carnevale A. Trisomy 9 mosaicism in a girl with multiple malformations. Ann Genet (Paris) 1990;33:165-8.

2 Warburton D. Editorial. Uniparental disomy: a rare consequence of the high rate of aneuploidy in human gametes. Am F Hum Genet 1988;42:215-6.

3 Temple IK, Cockwell A, Hassold T, Pettay D, Jacobs P. Maternal uniparental disomy for chromosome 14. F Med Genet 1991;28:511-4.

4 Schinzel A, Hayashi K, Schmid W. Mosaic trisomy and pericentric inversion of chromosome 9 in a malformed boy. pericentric inversion of chrom

5 Bowen P, Ying KL, Chung GSH. Trisomy 9 mosaicism in a newborn infant with multiple malformations. $\mathcal{f}$ Pediatr 1974;85:95-7.

6 Hall JG. How imprinting is relevant to human disease. Development 1990;(suppl):141-8.

7 Frydman M, Shabtai F, Halbrecht I, Elian E. Normal psychomotor development in a child with mosaic trisomy and pericentric inversion of chromosome 9. $\mathcal{f}$ Med Genet $1981 ; 18: 390-2$. 